\title{
Application of an Incisional Hernia Protocol at the Municipal Public Server Hospital of Sao Paulo, Brazil: A Retrospective Study
}

ISSN: 2637-7632

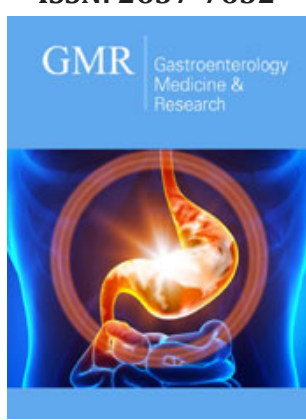

*Corresponding author: João Kleber de Almeida Gentile, Medical Professor of the Discipline of Digestive Surgery in University City of São Paulo (UNICID), Medical Doctor and Resident Physician, Department of Digestive Surgery, Public Servant Hospital Municipal (HSPM-SP), São Paulo (SP), Brazil

Submission: 海 August 24, 2021

Published: 䟧September 16, 2021

Volume 6 - Issue 2

How to cite this article: Caio Felicio de Oliveira, Paula Gombar, João Kleber de Almeida Gentile, et al. Application of an Incisional Hernia Protocol at the Municipal Public Server Hospital of Sao Paulo, Brazil: A Retrospective Study. Gastro Med Res. 6(2). GMR. 000632. 2021.

DOI: 10.31031/GMR.2021.06.000632

Copyright@ João Kleber de Almeida Gentile, This article is distributed under the terms of the Creative Commons Attribution 4.0 International License, which permits unrestricted use and redistribution provided that the original author and source are credited.
Caio Felicio de Oliveira ${ }^{1}$, Paula Gombar ${ }^{1}$, João Kleber de Almeida Gentile ${ }^{2 *}$, Renato Migliore ${ }^{2}$, Rodrigo Biscuola Garcia ${ }^{3}$, Pedro Marcos Santinho Bueno de Souza $^{3}$ and e José Cesar Assef ${ }^{4}$

${ }^{1}$ Medical Residend of the Discipline of Digestive Surgery, Public Servant Hospital Municipal (HSPM-SP), São Paulo (SP), Brazil

${ }^{2}$ Medical Professor of the Discipline of Digestive Surgery in University City of São Paulo (UNICID), Medical Doctor and Resident Physician, Department of Digestive Surgery, Public Servant Hospital Municipal (HSPM-SP), São Paulo (SP), Brazil

${ }^{3}$ Medical Doctor and Attending Physician, Department of Digestive Surgery, Public Servant Hospital Municipal (HSPM-SP), São Paulo (SP), Brazil

${ }^{4}$ Medical Doctor and Chief, Department of Digestive Surgery, Public Servant Hospital Municipal (HSPM-SP), São Paulo (SP), Brazil

\begin{abstract}
Background and Aim: Abdominal Incisional Hernias (AIH) are a frequent complication in medium incisions that lead to a decrease in life quality, socioeconomic losses and increased morbidity to those who have it. Self-care cost for patients evolving with AIH can increase from $97 \%$ up to $370 \%$ in the first 3 years postoperatively. Regarding this, the Digestive System Surgery Service of the Municipal Public Server Hospital's (HSPM) has created a protocol to deal with this type of patient since 2015, it has been applied up to the present moment. Incisional hernias result from a multifactorial process that includes preoperative factors such as the cessation of smoking, diabetes control, and obesity, such factors affect the proper functioning of the early stages of the healing process.

Patient and Method: Retrospective study with registry analysis of medical records and database records created on cloud regarding perioperative information and outcomes of 35 patients with complex incisional hernias treated under the protocol of patient care for incisional hernia on the Municipal Public Server Hospital (Attachment 1) by the Digestive System Surgery Service, between 2015 and 2019.

Result: In our analysis of 35 patients operated by the Digestive System Surgery Team in the period from 2015 to 2019, the average of hospital stay was 4.55 days per patient, with a total of $22.857 \%$ of complications, from seromas without serious repercussion to a wall infection which demanded debridement and reassessment for cutaneous flap. Onlay meshes were used in $57.14 \%$ of the cases, $37.14 \%$ were sublay meshes, and in two cases $(5.71 \%)$ meshes were placed in two different planes, sublay and onlay - sandwich technique. Of these cases, one was a lateral hernia by multiple procedures and the other was caused by a late postoperative of lumbotomy with lateral hernia evolution. There was a recurrence of a patient with a $6.9 \mathrm{~cm}$ hernia, he underwent two treatment attempts in 2005 and 2010, which also presented recurrence. In our service the patient was treated with the Rives-Stoppa technique. The patient chose not to undergo further treatment.
\end{abstract}

Conclusion: The protocol application to patients with AIH was associated with a recurrence rate of $2.86 \%$, in a universe of patients in which recurrence still remains a feared complication for both the surgeon and the patient. Furthermore, the complications were treated, most of them successfully, with clinical measures, except for a recurrence and a surgical wound infection in which debridement was necessary, adding up to $5.71 \%$.

Keywords: Hernia; Abdominal hernia; Incisional hernia; Ventral hernia

\section{Introduction}

Abdominal Incisional Hernias (AIH) are a frequent complication in medium incisions that lead to a decrease in life quality, socioeconomic losses and increased morbidity to those who have it [1]. Self-care cost for patients evolving with AIH can increase from $97 \%$ up to $370 \%$ 
in the first 3 years postoperatively [2]. Regarding this, the Digestive System Surgery Service of the Municipal Public Server Hospital's (HSPM) has created a protocol to deal with this type of patient since 2015, it has been applied up to the present moment.

Incisional hernias result from a multifactorial process that includes preoperative factors such as the cessation of smoking, diabetes control, and obesity, such factors affect the proper functioning of the early stages of the healing process [3]. Risk factors for AIH development can be divided into technical factors (incision type, suture choice, closure technique) or specific factors in each patient. Patient specific factors may be non-modifiable (prior laparotomy, chronic infection, malignancy) or modifiable (diabetes, obesity, use of immunosuppressive drugs, corticosteroids, tobacco consumption and malnourishment) [2].

The associations between procedures with medium incisions and the use of corticosteroids, immunosuppressive drugs, and smoking as factors of increased chance for complications are well established, especially on treatment of aortic aneurysms, diabetes and obesity [4,5]. Smoking increases the risk of infections, lung complications and surgical wounds. The cessation of smoking preoperatively reduces the risk of postoperative complications, rehospitalization and re-initiation [3]. Closing the abdominal wall with small bites is a well-accepted technique, such as the use of slow absorbing sutures and prophylactic mesh placement. These measures have been serving as a protection factor for the development of AIH2. The pathophysiology of the wall defect is associated with inflammatory changes that mainly affect the proportion between collagen type 1 and collagen type 3 leading to higher incidence of defects on the extracellular matrix [1].

In this sense, the use of steroids or immunosuppressive drugs has shown an increased risk of recurrence of incisional hernia after laparotomic repair with hernia correction using rives-stoppa technique, since it increased the incidence of bruises and the need for reassessment in a retrospective study with 213 patients [6]. Furthermore, the history of surgical site infection being a risk factor in the first 30 postoperative days is associated with new episodes of postoperative surgical site infection for new infections, there is need for new procedures and for non-programmed admission [7]. A retrospective study of 14,290 patients resulted in a $48 \%$ incidence of AIH development in the first postoperative year, 33\% in the second year and 19\% in the third year. Taking into account established risk factors for poor evolution of $\mathrm{AIH}$ treatment, a protocol for taking care of the patient with incisional hernia was created at the Digestive System Surgery Service of the HSPM and applied since 2015 to its patients (annex 1). The present project consists of evaluating the outcome of the patients treated between 2015 and 2018 under this protocol.

\section{Objective}

To evaluate the application of the protocol on patients with incisional hernia treated at the Digestive System Surgery Service of the HSPM.

\section{Methodology}

Retrospective study with registry analysis of medical records and database records created on cloud regarding perioperative information and outcomes of patients with complex incisional hernias treated under the protocol of patient care for incisional hernia on the Municipal Public Server Hospital (Attachment 1) by the Digestive System Surgery Service, between 2015 and 2019. Items to be evaluated: 1)hospital stay, 2)surgical wound infection, 3)relevant complications, 4)mesh position

\section{Result}

In our analysis of 35 patients operated by the Digestive System Surgery Team in the period from 2015 to 2019, the average of hospital stay was 4.55 days per patient, with a total of $22.857 \%$ of complications, from seromas without serious repercussion to a wall infection which demanded debridement and reassessment for cutaneous flap. Onlay meshes were used in $57.14 \%$ of the cases, $37.14 \%$ were sublay meshes, and in two cases $(5.71 \%)$ meshes were placed in two different planes, sublay and onlay - sandwich technique. Of these cases, one was a lateral hernia by multiple procedures and the other was caused by a late postoperative of lumbotomy with lateral hernia evolution.

There was a recurrence of a patient with a $6.9 \mathrm{~cm}$ hernia, he underwent two treatment attempts in 2005 and 2010, which also presented recurrence. In our service the patient was treated with the rives-stoppa technique. The patient chose not to undergo further treatment. Two patients developed chronic postoperative pain, gabapentin was used to treat one of the cases and prolonged simple analgesia to treat the other. Other complications observed were symptomatic seroma, retroperitoneal hematoma and operative wound dehiscence, all of them had adequate resolutions from clinical measures. There was no mortality in the group studied. Table 1 shows mesh placement division on correcting hernias. Table 2 shows absolute numbers and percentages of events.

Table 1: Mesh placement division on correcting hernias.

\begin{tabular}{|c|c|c|}
\hline Onlay & Sublay & Onlay + Sublay \\
\hline $20(57,142 \%)$ & $13(37,14 \%)$ & $02(5,714 \%)$ \\
\hline
\end{tabular}

Table 2: Absolute numbers and percentages of events.

\begin{tabular}{|c|c|c|}
\hline Post-Operative Events & $\mathbf{n}$ & $\mathbf{\%}$ \\
\hline Symptomatic seroma & 1 & 2,85 \\
\hline $\begin{array}{c}\text { Post-operative chronic } \\
\text { pain }\end{array}$ & 2 & 5,71 \\
\hline Scrotal hematoma & 1 & 2,85 \\
\hline $\begin{array}{c}\text { Surgical wound } \\
\text { infection }\end{array}$ & 2 & 5,71 \\
\hline Dehiscence & 1 & 2,85 \\
\hline Recurrence & 1 & 2,85 \\
\hline Deaths & 0 & 0 \\
\hline Total & 8 & 22,85 \\
\hline
\end{tabular}




\section{Attachment 1}

Protocol of treatment to patients with incisional hernia on the digestive system surgery service of the municipal public server hospital of Sao paulo, Brazil

Incisional hernia represents a failure in the early stages of inflammatory reaction of the healing process. Diseases like diabetes and comorbidities such as smoking decrease the recruitment of inflammatory cells. The prevention of Abdominal Incisional Hernia (AIH) should aim at maintaining the appropriate inflammatory reaction in the early stages of healing.

Wall closure: The wall closure in both elective and emergency operations must follow the 'small bite' guidelines, with continuous stitches, using slow absorption wires maintaining $3 \mathrm{~mm}$ to $6 \mathrm{~mm}$ (mean of $05 \mathrm{~mm}$ ) distance from wound edges as well as between plications.

Prophylactic mesh (onlay): The use of prophylactic onlay mesh in emergencies prevents hernias without increasing global comorbidities, even in the presence of infection. Regarding abdominal aortic aneurysm repair operations, the use of the mesh is related to a lower rate of incisional hernia without increased chances of complications.

Paracolostomic hernias: The use of meshes to prevent paracolostomic hernias was efficient in preventing paracolostomic defects. However, there was no adherence to its use by surgeons because of the risk of more severe complications, even though these were not prevalent.

Hernia risk predictors: Obesity, open surgery, smoking, previous surgery, abdominal aortic surgery and contamination are factors that increase the risk of AIH. Measures that affect these factors are crucial in treating AIH to mitigate recurrences. Control of BMI $\left(<30 \mathrm{~kg} / \mathrm{m}^{2}\right)$, cessation of smoking, adequate glycemic control, adequate wall closure techniques (small bites), and connective tissue reinforcement should be practiced in patient care for AIH prevention and treatment.

AIH prevention: Intraoperative temperature control increased collagen deposition and lowered infection rates, but with poor evidence. $20-10 \mathrm{~mm}$ wide prosthesis applied in the closure of incisions with technique of small bites improve the chances of $\mathrm{AIH}$ and should be used even in the presence of infections and in emergencies.

\section{Preoperative guidelines}

Obesity: Patients should have a BMI below $30 \mathrm{~kg} / \mathrm{m}$. Patients between 30 and $35 \mathrm{k} / \mathrm{m}^{2}$ who have failed clinical treatment for weight loss should be advised to carry out a liquid diet for ten days just before the operation date, being weighed in the 1st and 10th days. If there is no weight loss, the operation must be canceled. Patients with BMI> 35 will not be operated.

Glucose: All diabetic patients should have adequate glycemic control with glycated hemoglobin $<6.5$ in the last three months.
Smoking: Only patients who have not consumed tobacco for 90 days will undergo surgery.

Emergencies: Patients in reoperations performed by the Digestive System Surgery Service of the HSPM, even in the presence of infection, should have a wall closure with a continuous suture technique using a slow absorption wire with a mean distance between plications of $5 \mathrm{~mm}$. An onlay mesh $10-20 \mathrm{~mm}$ wide should be placed to reinforce this suture.

Image exams: All complex hernias should be evaluated with digital tomography without contrast with the patient in Valsalva (wall protocol).

\section{Postoperative guidelines}

Diet: Aim to reintroduce a diet that progresses according to its density (from liquid to general) according to the patient's acceptance. To do so, stimulate early ambulation.

Analgesia: Maintain simple analgesia (dipyrone 1g 6/6hours/ paracetamol $500 \mathrm{mg} 8 / 8 \mathrm{hours}$ ) schedule for all patients and evaluate medication withdrawal according to improved pain control from the second postoperative day onwards. Do not use NSAIDs for patients over 60 years-old or patients with any degree of chronic kidney damage. Avoid use of tramadol because of its association with episodes of vomiting. If severe pain occurs, administer morphine $2 \mathrm{mg}$ 4/4hours. Patients with reoperation who have a history of chronic pain may need to use gabapentin 300mg up to 3 times a day for 3 months after surgery, with medication withdrawal evaluation done in ambulatory. These individuals should be followed up by the neurology ambulatory clinic for chronic pain management.

Drainage: Patients with extensive subcutaneous tissue detachment should have dead spaces drained with suction drains.

\section{Thrombosis prophylaxis}

High surgical compression stockings $3 / 4$ (white) should be used intraoperatively and during hospitalization.

Low-molecular-weight heparin should be introduced 08hours after the end of surgery. (enoxaparin at a dose of $1 \mathrm{mg}$ for every patient body weight kg every 24hours with a maximum dose of $120 \mathrm{mg} /$ day). The non-use this prophylaxis is justified only if there is a sign of bleeding (open drainage, gastrointestinal tract bleeding).

Antibiotic therapy $\mathbf{x}$ antibiotic prophylaxis: Patients with complex hernias should maintain therapeutic use with $1^{\text {st }}$ generation cephalosporin (cefadroxil 500mg every 12hours for 0714 days). Patients with simple hernias should have prophylactic use with the same class of antibiotics for 24 hours.

Prophylaxis of stress ulcers: PPIs should be used for all patients because of increased risk of stress gastric ulcer due to surgical trauma, use of NSAIDs and low molecular weight heparins.

\section{FO ambulatory evaluation}

We contraindicate blind drainage in the HSPM hernia ambulatory because of the risk of seroma below prostheses and 
inadvertent lesions of intraabdominal elements. Always evaluate with imaging exams (ultrasonography or tomography). Look for changes resulting from chronic medications and/or allergies, as well as control of capillary glycemia and insulin according to capillary glycemia (Table 3).

Table 3: Prescription model POI.

\begin{tabular}{|c|c|}
\hline 1 & Light diet when well awake \\
\hline 2 & Cefazolin $1 \mathrm{~g}$ every 12 hours (EV) \\
\hline 3 & Dipyrone 1g every 6 hours (EV) \\
\hline 4 & Ketoprofen 50mg every 12 hours (EV) \\
\hline 5 & Omeprazole $40 \mathrm{mg}$ once early in the morning (EV) \\
\hline 6 & Ondansetron $4 \mathrm{mg}$ every 8 hours if nauseous or vomiting \\
\hline 7 & $\begin{array}{c}\text { Enoxaparin 40mg every } 24 \text { hours after } 08 \text { hours from the } \\
\text { end of anesthesia }\end{array}$ \\
\hline 8 & (Morphine $2 \mathrm{mg}+\mathrm{SF} 0,9 \% 8 \mathrm{~mL}$ ) if in severe pain (EV) \\
\hline 9 & High elastic compression stockings \\
\hline 10 & Abdominal belt \\
\hline 11 & Respiratory physiotherapy \\
\hline 12 & Respiron ${ }^{\circledR}$ ( 03 sets of 10 aspirations every 3 hours) \\
\hline 13 & Stimulate ambulation \\
\hline
\end{tabular}

\section{Discussion}

The data collected in this study shows a below average number of adverse events and complications after AIH corrections.

We consider as factors that contributed to these indices, especially regarding recurrence and wound infection:

1) Adherence to the protocol by the service and by the patients - smoking abstinence and weight control;

2) The underdiagnosis of surgical wound infection due to the fact that it is a retrospective study, being susceptible to failures in the registry of medical records; and

3) the use of antibiotics until the $7^{\text {th }}$ postoperative day. Regarding seromas, we credit their low incidence numbers to the use of Baroudi stitches and drainage in most of the cases.

\section{Conclusion}

The protocol application to patients with AIH was associated with a recurrence rate of $2.86 \%$, in a universe of patients in which recurrence still remains a feared complication for both the surgeon and the patient. Furthermore, the complications were treated, most of them successfully, with clinical measures, except for a recurrence and a surgical wound infection in which debridement was necessary, adding up to $5.71 \%$. Therefore, preoperative evaluation looking for the control of variables (comorbidities proven to be detrimental to the outcome, operative technique, analgesia, infection prevention, etc.) brought adequate results for patients treated at the HSMP Digestive System Surgery Service.

\section{Conflict of Interest}

There was no conflict of interests between the project's authors and external influences.

\section{References}

1. Harris HW, Hope WH, Adrales G, Andersen DK, Deerenberg EB, et al. (2018) Contemporary concepts in hernia prevention: Selected proceedings from the 2017 international symposium on prevention of incisional hernias. Surgery 164(2): 319-326.

2. AlliVV,ZhangJ, Telem DA (2018) Impact of incisional hernia development following abdominal operations on total healthcare cost. Surg Endosc 32(5): 2381-2386.

3. DeLancey JO, Blay Jr E, Hewitt DB, Engelhardt K, Bilimoria KY, et al. (2018) The effect of smoking on 30-day outcomes in elective hernia repair. Am J Surg 216(3): 471-474.

4. Wiegering A, Liebetrau D, Menzel S, Bühler C, Kellersmann R, et al. (2018) The incidence of incisional hernia after aortic aneurysm is not higher than after benign colorectal interventions. Gefässchirurgie 23(1): 23-31.

5. Bosanquet DC, Ansell J, Abdelrahman T, Cornish J, Harries R, et al. (2015) Systematic review and meta-regression of factors affecting midline incisional hernia rates: Analysis of 14618 patients. PLoS One 10(9): e0138745.

6. Bueno-Lledó J, Torregrosa A, Jiménez-Rosellón R, García P, Bonafé S, et al. (2019) Predictors of hernia recurrence after Rives-Stoppa repair in the treatment of incisional hernias: A retrospective cohort. Surg Endosc 33(9): 2934-2940.

7. Tastaldi L, Petro CC, Krpata DM, Alkhatib H, Fafaj A, et al. (2019) History of surgical site infection increases the odds for a new infection after open incisional hernia repair. Surgery 166(1): 88-93. 